\title{
Optimal design of conductive and inductive charging system for bus rapid transit network
}

\author{
Adedayo Asaolu \\ Electronic and Electrical Engineering \\ University of Strathclyde \\ Glasgow, United Kingdom \\ adedayo.asaolu@strath.ac.uk
}

\author{
Stuart Galloway \\ Electronic and Electrical Engineering \\ University of Strathclyde \\ Glasgow, United Kingdom \\ stuart.galloway@strath.ac.u
}

\begin{abstract}
This paper proposes a multi-terminal allocation of charging infrastructure for the public bus transit network that integrates both inductive and conductive priority charging to ensure energy management without disrupting the operational bus schedule. The charging model is formulated as a mathematical optimisation problem that allocates multipriority chargers for the transit network. The formulated optimisation problem is solved using a particle swarm optimisation (PSO) algorithm. The model is validated using Lagos BRT data. The results indicate that the integration of both inductive and multi-terminal conductive priority chargers in the transit system can serve as an energy management system and eliminate charging dwell time.
\end{abstract}

Keywords - Battery-electric bus (BEB), conductive charger inductive charger, PSO, Transit network.

\section{NOTATION}

\begin{tabular}{|c|c|}
\hline$C h^{\text {Select }}$ & Charging priority selector \\
\hline$d_{\text {ind }}^{\text {trav }}$ & Length of an inductive charger \\
\hline$E_{b}^{\text {arr }}, E_{\text {ind }}^{\text {arr }}$ & $\begin{array}{l}\text { Energy capacity of the BEB on arrival at the terminal/at } \\
\text { the start of inductive charging }\end{array}$ \\
\hline$E_{b}^{c a p}$ & BEB battery energy capacity \\
\hline$E_{b}^{c h}$ & Energy recharged via conductive charging \\
\hline$E_{\text {ind }}^{\text {gain }}$ & Energy gain from inductive charging \\
\hline$E_{b}^{\max }, E_{b}^{\min }$ & Max, min Energy capacity of the BEB \\
\hline$E_{b}^{i, i+n}$ & Energy req. to travel from terminal $i$ to $i+n$ \\
\hline$E_{\text {ind }}^{\text {rec }}$ & Energy received via inductive charging \\
\hline$E_{\text {ind }}^{\text {une }}$ & Energy used to travel the inductive cable \\
\hline$N_{c h}^{c o n d}$ & Number of conductive chargers \\
\hline$N_{c h}^{i n d}$ & Number of inductive chargers \\
\hline$N_{\text {ch total }}^{\text {tot }}$ & Total number of chargers \\
\hline$N_{c h}^{l}, N_{c h}^{m}, N_{c h}^{h}$ & $\begin{array}{l}\text { Number of low, medium and high priority } \\
\text { conductive chargers }\end{array}$ \\
\hline$P_{c h}^{c o n d}, P_{c h}^{i n d}$ & Power of conductive and inductive chargers \\
\hline$P_{(t)}^{i}$ & Power used by inductive charger over time interval ( $\mathrm{t}$ ) \\
\hline$P_{(t)}^{l}, P_{(t)}^{m}, P_{(t)}^{h}$ & $\begin{array}{l}\text { Power used by low, medium and high priority conductive } \\
\text { charger over time interval }(\mathrm{t})\end{array}$ \\
\hline$P_{(\max )}^{l}, P_{(\operatorname{ma}}^{m}$ & $\begin{array}{l})_{1}, P_{(\max )}^{h} \text { Max. power capacity low, medium and high } \\
\text { priority conductive charger }\end{array}$ \\
\hline$P_{d s}$ & Power used by BEB per time step \\
\hline$P_{\text {use }}$ & Power used by BEB when moving over inductive cable \\
\hline$P_{(t)}^{\text {total }}$ & Power used by all the chargers in the transit with time \\
\hline$t_{b}^{a r r}$ & Arrival time of the BEB at the bus terminal \\
\hline$t_{b}^{c h}$ & BEB conductive charging duration \\
\hline$t_{b}^{s}$ & The time the BEB is scheduled for the next trip $\mathrm{i}$ to $\mathrm{i}+\mathrm{n}$ \\
\hline$t_{\text {ind }}^{\text {trav }}$ & Travelling time over induction cable \\
\hline$t_{b}^{\text {wait }}$ & Waiting time of the BEBs at the terminals \\
\hline$t_{b}^{i, i+n}$ & Travel time from terminal $i$ to $i+n$ \\
\hline
\end{tabular}

Petroleum Technology Development Fund, Abuja Nigeria, under grant number $\mathrm{PTDF} / \mathrm{ED} / \mathrm{PHD} / \mathrm{AAA} / 1085 / 17$, sponsors the research work.

\author{
B. Binary Variables \\ $\propto^{l}, \propto^{m}, \propto^{h}$ : Binary variable for low, medium and high priority charging \\ $\beta \quad$ Binary variable for inductive charging
C. Indices
$i, j, n \quad$ Indices for terminal, BEB and terminal position
in the transit networks
$t, T \quad$ Indices for time steps
$b, B \quad$ Indices for transit network battery electric buses (BEBs)

\section{INTRODUCTION}

Generally, developed countries are planning, and implementing schemes for reducing greenhouse gas emissions, and the critical focus is the electrification of the transport system (both public and private vehicles). However, in the developing countries, the electrification of public transport and transit network is probable to evolve ahead of individual owned vehicle because it can be controlled by government policy and deliberate investment in public transport. Importantly, the power infrastructure for public transport fleets is different from that for consumers as the charging infrastructures for the electric public transport system are more of hub base [1] (i.e., terminals, deports and bus-stops) that can be power with renewable energy mini-grids. Currently, in major European cities, 40$60 \%$ of trips are achieved via sustainable means. In developing countries cities such as Lagos (Nigeria) that have a population of about 21 millions people [2], this is compounded. The characteristics of the transport system in Lagos include the unavoidable daily experience of the worst traffic situation due to road congestion and GHG emission that are predominantly from automobiles [3]. Presently, Lagos has a bus rapid transit (BRT) system that connects people from the suburbs to the central business district [4]. The greening of this public asset, in combination with favourable GHG solutions, is an attractive option for policymakers to eliminate 'driveway' congestion and support the adoption of EV.

The development and adoption of Battery-Electric Buses (BEBs) have been increasing rapidly within the last decade. These vehicles offer zero-emission, quiet operation, high efficiency, reliability, and better acceleration compared to traditional buses. In comparison to trolley bus technology, they eliminate the need for constant grid connection, and the transit route can be modified without infrastructure change, and the cost of ownership is lower than diesel buses [5].

In [6], the comparative on-road evaluation of diesel buses with battery-electric buses BEBs is examined in a broad range of circumstances. In this work, the BEBs cuts 
petroleum use by $85-87 \%$ in comparison to a diesel bus and attains a $32-46 \%$ decrease in the use of fossil fuel and 19$35 \%$ in $\mathrm{CO} 2$ emissions while evaluating from a life-cycle perspective.

Nonetheless, the charging systems that support long driving distance are still in the early phases of development and very expensive. Moreover, the schedule for BEBs needs to consider a variety of parameters about the energy system [5],[7]. Mitigating these issues while observing the operational bus schedules, the adequate provision for the trips demand energy needs to be considered. In the literature, different strategies have been proposed, and the common theme includes charging and battery swapping [8] [9] and repurposing infrastructure [11]. Under a battery swapping system, the BEB can replace its depleted battery pack with a full one within a few minutes. Then the drain battery packs are recharged at the station and later swapped for other arriving BEBs [8][10]. However, the battery swapping system requires a significant extra investment on the batteries and its depot [11]. Hence, the majority of the research work in BEBs have been concentrating on the optimal sizing of battery and charging infrastructures to satisfy the BEBs transit operation plan [12]-[14].

As far as these authors are aware, previous research work has not considered:

1. The application of opportunity charging with the integration of renewable energy resources for a bus transit system while taking note of the peculiarity of the cities in the developing countries such as the situation in Lagos, Nigeria where the grid is weak, and traffic congestion is beyond problematic levels.

2. The use of both the conductive and inductive charging system in a transit network to reduce the operational downtime as a result of BEB charging duration.

3. The multi-terminal allocation of charging infrastructure for a bus transit system.

4. The priority charging method that ensures effective energy management at the conductive charging terminals.

In this work, the transit electrification system considered is the multi-terminal allocation of charging infrastructure for a bus transit system where both conductive (priority) and the inductive charging systems are employed. Such a system is developed to manage the system energy demand and ensure that the operational service is reliable by optimising the charging station infrastructure: charger operating capacity and the numbers of chargers. The model developed for this work is widely applicable; the BRT network in Lagos, Nigeria, is used as a case study.

The remainder of this paper is arranged as follows: Section II is the description of the charging model. In section III, the optimisation model is presented. Section IV presents the numerical analysis that describes the simulation result with case studies that test the effectiveness of the proposed optimisation model. The conclusion and future work are given in section $\mathrm{V}$.

\section{Charging MOdel}

\section{A. Model Description}

The process flow diagram in fig 1 illustrates the generic terminal-based simulation model proposed in this work. The charging that occurs at the BEB terminals is assigned based on the priority that depends on the state of charge of the BEB battery on arrival at the terminal.

The long routes (routes $>10 \mathrm{~km}$ ) within the transit network are proposed to have inductive chargers; for simplicity, they are located at the middle of the trip. This inductive charging stage occurs when the bus is either moving slowly or is stopped for a few minutes to pick up and drop off passengers. The BEB that can be charged inductively will contain additional equipment installed that acts a 'pickup' device for collection of electrical energy from the installed on-road power transmitters. This 'scavenged' charging will enable topping up of the BEBs batteries to extend their range and protect the BEBs batteries from overdrain.

In this work, a similar modelling approach to that presented in [12] [13] has been adopted. While the focus in these leading research articles was to consider infrastructure requirements and grid effects respectively, here, a priority charging scheme has been included, the option for 'on-thefly' inductive charging on longer routes made available and the context of the Lagos BRT accounted for.

The process flow diagram for the 'modelled system' shown in fig 4 is described as follows:

1. Each BEB is operating on a fixed route within the BRT network; this fixed route has a terminal at both ends where the BEB can use conductive chargers. The routes that are longer than $10 \mathrm{~km}$ have midway inductive chargers. In addition, there are multiple bus stops to load and unload passenger along each route.

2. Each BEB is equipped with one battery unit denoted with $\left(E_{b}^{c a p}\right)$ in $\mathrm{kWh}$, and all the BEB batteries are of the same size. In this work, the BEBs batteries have a minimum $\left(E_{b}^{\text {min }}\right)$ and maximum $\left(E_{b}^{\max }\right)$ range of the total battery energy capacity.

3. The arrival time $\left(t_{b}^{\text {arr }}\right)$, the battery energy capacity on arrival $\left(E_{b}^{a r r}\right)$ and the battery capacity $\left(E_{b}^{c a p}\right)$ are logged on arrival at the BEB terminal. The duration between the arrival and the next schedule is called stay time which is the maximum time allowed for BEB charging

$$
\begin{aligned}
& t_{b}^{\text {wait }}=t_{b}^{S}-t_{b}^{a r r} \\
& t_{b}^{\text {ch }} \leq t_{b}^{\text {wait }}
\end{aligned}
$$

4. Then, the energy consumption of the BEB for the next scheduled trip from terminal $i$ to $i+n$ is given as:

$$
E_{b}^{i, i+n}=\int_{t=i}^{t_{i+n}} P_{d s} d t
$$

Where ' $n$ ' is an integer indicating the subsequent number of terminal's until the end of the scheduled trip.

5. If the $E_{b}^{a r r}$ satisfies the next schedule trip of the BEB energy demand, the model will further validate that the $E_{b}^{\text {min }}$ constraint is satisfied. If the $E_{b}^{\text {min }}$ constraint is also satisfied the charging of the BEB depends on the availability of the charging unit before the next schedule $t_{b}^{s}$ and the BEB charging in this circumstance is given low-priority.

6. However, if the $E_{b}^{\text {min }}$ constraint is not satisfied, the model verifies if the next scheduled route has a midway 
inductive charger that can top-up the BEB battery to satisfy the $E_{b}^{\text {min }}$ constraint. With the availability of inductive charger along the route, the BEB is scheduled to charge at a low priority (that depends on the availability of charging unit before the next scheduled trip). Otherwise, the BEB is allocated to be charge at a medium priority.

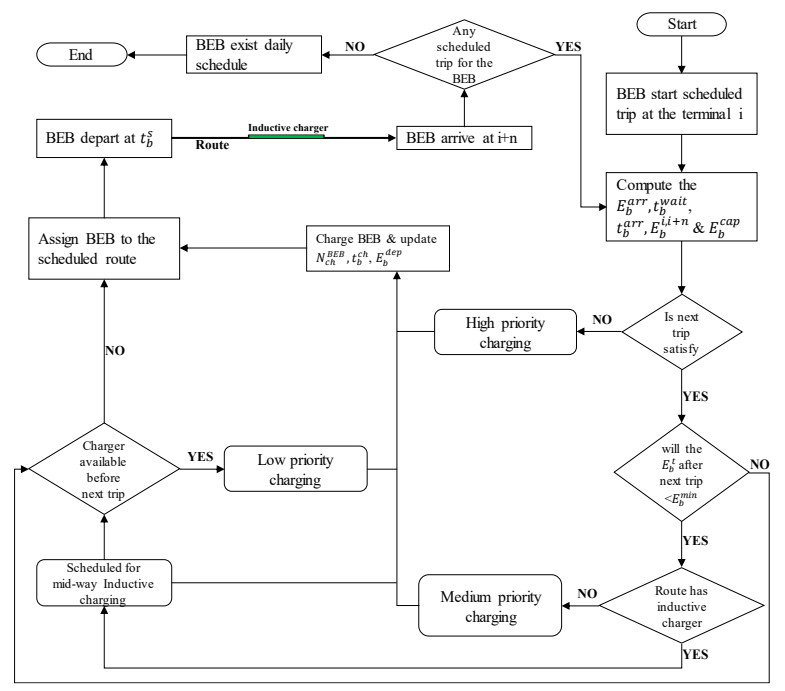

Fig 1: Flow chart for the terminal-based charging model

7. If the $E_{b}^{a r r}$ cannot meet the energy consumption demands of the next scheduled trip $i$ to $i+n$, the BEB is allocated for charging with high priority charging.

Thus, on the arrival of BEB at the terminal, the charging priority is selected to be high, medium and low is expressed in the form of energy dynamics as follows:

$$
C h^{\text {select }}=\left\{\begin{array}{cr}
\text { High } & E_{b}^{a r r} \leq E_{b}^{i, i+n} \\
\text { Medium } & E_{b}^{\text {arr }}-E_{b}^{i i+n} \leq E_{b}^{\min } \\
\text { Low } & \text { otherwise }
\end{array}\right\}
$$

Where $C h^{\text {select }}$ is the charging priority selector. $E_{b}^{i, i+n}$ is the energy required for the BEB next scheduled trip from the terminal $i$ to $i+n$. The nomenclature of high, medium and low correspond to high, medium and low charging priority respectively

\section{B. Model Assumptions}

The Lagos BRT BEBs example considered in this work uses a fixed dedicated route that is called Transit-Way. The Transit-Way creates dedicated corridors for the buses that are separated from other traffic with concrete walls as shown in fig 4.

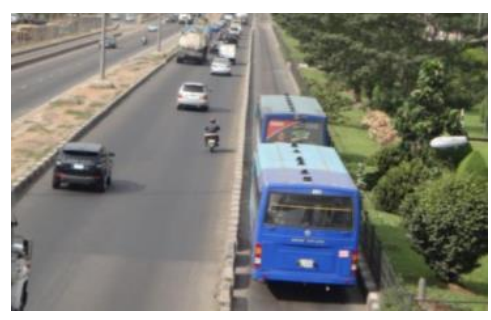

Fig 2: BRT route with buses

For modelling simplicity, it is assumed that the scheduled time for the transit is accurate and the power demand is based on the travelling time between various named staging points and generally as points $i$ to $i+n$, and the travelling time $t_{b}^{i, i+n}$ is taken as the bus schedule duration to travel from terminal $i$ to $i+n$. In this work, the rate of energy consumption of the BEBs is assumed to be $1.34 \mathrm{kWh} / \mathrm{km}$, which is the value evaluated for the opportunity charging strategy in the relevant literature [13].

\section{OPTIMISATION MODEL}

\section{A. Objective Function}

The objective is to meet the infrastructure requirements while minimising the total number of charging facilities. On this basis, the objective function for the optimal number of chargers in the electric transit network is express as:

$$
\begin{aligned}
\operatorname{Min} \sum_{b \in B} \sum_{t \in T}\left(N_{c h}^{l} \cdot P_{(t)}^{l} \cdot \propto^{l}+N_{c h}^{m} \cdot P_{(t)}^{m} \cdot \propto^{m}\right. \\
\left.+N_{c h}^{h} \cdot P_{(t)}^{h} \cdot \propto^{h}\right)+N_{c h}^{i} \cdot P_{(t)}^{i} \cdot \beta
\end{aligned}
$$

Here the first (bracketed) term in (5) represents charging capability associated priorities of the fixed infrastructure at BEB terminals while the second term accounts for the number of the inductive transmitter in the transit network. The duration for the inductive charging is represented with $t_{c h}^{i n d}$, which is equivalent to the time for the BEB to travel distance $d_{\text {ind }}^{\text {trav }}$ as shown in fig 3 .

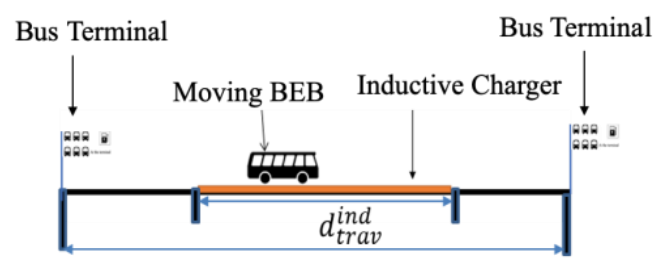

Fig. 3: Model of two-transit terminal

\section{B. Constraints}

At the $i^{\text {th }}$ terminal the binary variable $\propto^{l}, \propto^{m}$ and $\propto^{h}$ have to ensure that a BEB can only charge using only one of the charging priority state in equation (4), where

$$
\begin{gathered}
\propto^{l}= \begin{cases}1 & 0 \leq P^{\iota} \leq P_{\text {max }}^{l} \\
0 & \text { otherwise }\end{cases} \\
\propto^{m}= \begin{cases}1 & P_{\text {max }}^{l} \leq P^{m} \leq P_{\text {max }}^{m} \\
0 & \text { otherwise }\end{cases} \\
\propto^{h}= \begin{cases}1 & P_{\text {max }}^{l} \leq P^{h} \leq P_{\text {max }}^{h} \\
0 & \text { otherwise }\end{cases} \\
\propto^{l}+\propto^{m}+\propto^{h}=1 \quad \propto \in[0,1]
\end{gathered}
$$

The summation of the energy used by all BEBs that charge at a particular terminal can be described as follows:

$$
\begin{gathered}
\sum_{j=1}^{m} \sum_{i=1}^{n} E_{b}^{i, i+n} \leq P_{(t)}^{\text {total }} \\
\sum_{t \in T} P_{(t)}^{l}+P_{(t)}^{m}+P_{(t)}^{h}+P_{(t)}^{i}=P_{(t)}^{\text {total }}
\end{gathered}
$$

When the BEB arrives at any of the designated BEB terminals, and it receives a charge the constraint is given as: 


$$
\begin{aligned}
& E_{b}^{a r r}+\int_{t=0}^{t=t_{b}^{c h}} P_{c h}^{\text {cond }} d t \leq E_{b}^{\max } \\
& E_{b}^{a r r}+E_{b}^{c h} \leq E_{b}^{\max }
\end{aligned}
$$

In a route that is equipped with an inductive charging system (as shown in fig 5) the BEB battery energy level after the travelling distance $d_{\text {ind }}^{\text {trav }}$ is expressed as:

$$
\frac{d E\left(t_{\text {ind }}^{\text {trav }}\right)}{d t}=-P_{u s e}\left(t_{\text {ind }}^{\text {trav }}\right)+P_{c h}^{\text {ind }}\left(t_{\text {ind }}^{\text {trav }}\right)
$$

Where $P_{u s e}\left(t_{\text {ind }}^{\text {trav }}\right)$ is the rate of consumption when the BEB is travelling the distance $d_{\text {ind }}^{\text {trav }}$ and $P_{c h}^{\text {ind }}\left(t_{\text {ind }}^{\text {trav }}\right)$ is the energy supply to the battery at the time $t_{\text {ind }}^{\text {trav }}$ that is required to travel distance $d_{\text {ind }}^{\text {trav }}$.

$$
\begin{aligned}
E_{\text {ind }}^{\text {arr }}-\int_{t=0}^{t=t_{\text {ind }}^{\text {trav }}} & P_{\text {use }} d t+ \\
& \int_{t=0}^{t=t_{\text {ind }}^{\text {trav }}} P_{c h}^{\text {ind }} d t \leq E_{b}^{\max }
\end{aligned}
$$

Rewriting equation (15) in the form of energy dynamics gives

$$
\begin{gathered}
E_{\text {ind }}^{\text {arr }}-E_{\text {ind }}^{\text {use }}+E_{\text {ind }}^{r e c} \leq E_{b}^{\text {max }} \\
E_{\text {ind }}^{\text {gain }}=E_{\text {ind }}^{r e c}-E_{\text {ind }}^{\text {use }} \\
E_{\text {ind }}^{\text {use }} \leq E_{\text {ind }}^{\text {gain }} \\
E_{\text {ind }}^{\text {gain }} \leq P_{(t)}^{i} \\
\frac{t_{\text {ind }}^{\text {trav }}}{t_{\text {ind }}^{\text {ch }}} \leq \beta \quad \beta \in[0,1]
\end{gathered}
$$

$\beta$ is one when route $\mathrm{i}$ to $\mathrm{i}+1$ has an inductive charger, and otherwise is zero.

The total chargers in the transit system is given as

$$
\begin{gathered}
N_{c h}^{\text {total }}=N_{c h}^{\text {cond }}+N_{c h}^{i} \\
N_{c h}^{\text {cond }}=N_{c h}^{l}+N_{c h}^{m}+N_{c h}^{h}
\end{gathered}
$$

\section{NUMERICAL ANALYSIS}

In this work, a particle swarm optimisation (PSO) algorithm is applied to find the optimal number of chargers for the multi-terminal transit system while considering an objective function (5) and the constraints expressed from equation (6) to (11), (19) and (20). The particle swarm optimisation (PSO) is used as the solution algorithm because it is very robust for solving complex nonlinear optimisation problems. PSO is a metaheuristic algorithm [16]. Further details on modelling and applications of PSO algorithm can be found in [15]-[17]. The PSO solution algorithm is modelled in MATLAB 2019b and runs on a Windows ten computer with core i5, 3.2GHZ processor, and 16G RAM.

\section{A. Case Study Description}

Table I presents the summary data for the six bus terminals of Lagos BRT. The data gives a summary of the number of buses, average trip time, and the mean daily trip time per bus per terminal in minutes. The multi-terminal characteristic of the Lagos BRT is shown in fig.4. From the figure, the total distance of the transit network is about $35 \mathrm{~km}$, and the maximum allowable assumed battery capacity of the BEB is $120 \mathrm{~kW}$. In this work, the long routes within the transit are equipped with inductive chargers, as described in section II. The Lagos bus transit network has six terminals, as shown in figure 4 . In this model, the inductive charger is configured as a fixed parameter that is located between terminal 2 and 3 that has a distance of $13 \mathrm{~km}$, terminal 3 , and 4 that has a distance of $12.5 \mathrm{~km}$ and terminal 4 and 5 that has a distance of $10.4 \mathrm{~km}$.

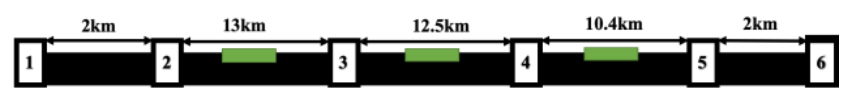

$16 \quad \begin{aligned} & \text { The bus terminals and the proposed } \\ & \text { location of the induction charging system }\end{aligned}$

Fig. 4: Model of the Lagos transit network

The allocation of the inductive charging infrastructures is considered reasonable because these routes are the longest in the transit. All are on the major trunk road that links all the BEBs terminals traversing Lagos. All the inductive chargers are configured to have the same capacity of $500 \mathrm{~kW}$, and the length of the inductive cable is assumed about 100 meters. These inductive charges are proposed to be located near major bus stops. Because the vehicles slow down as they approach the stations and then eventually stop to offload and load passengers - these allow a short but sufficient time to 'top-up' the BEB battery and several buses can be charged simultaneously.

Table I: Transit Data Summary

\begin{tabular}{|l|c|c|c|c|c|c|}
\hline \multirow{2}{*}{\multicolumn{1}{|c|}{ Variable }} & \multicolumn{7}{|c|}{ Terminals } \\
\cline { 2 - 7 } & $\mathbf{1}$ & $\mathbf{2}$ & $\mathbf{3}$ & $\mathbf{4}$ & $\mathbf{5}$ & $\mathbf{6}$ \\
\hline No. of BEB & 48 & 40 & 45 & 47 & 40 & 45 \\
\hline Mean trip time & 57 & 50 & 39 & 38 & 47 & 48 \\
\hline Mean daily trip time & 684 & 600 & 468 & 456 & 564 & 576 \\
\hline
\end{tabular}

The conductive chargers at the terminals are designed with various capacities, and these capacities are configured to be high, medium and low priority charging as described section II. In this work, the high priority chargers are configured to have the maximum capacity of $400 \mathrm{~kW}$, the medium-capacity chargers have the maximum capacity of $200 \mathrm{~kW}$, and the low capacity chargers have the capacity of $50 \mathrm{~kW}$.

\section{B. Simulation studies}

The simulation result is shown in Table II. indicates that 24 conductive multi-terminal chargers of various capacity should be allocated to the transit network. The bus terminal one has an optimal allocation of 6 - with 2 configured to be high (H), 1 medium (M) and 3 low (L) capacity chargers and so on for the remaining terminals.

Table II: Simulation result for priory charging

\begin{tabular}{|l|c|c|c|c|c|c|c|}
\hline \multicolumn{2}{|c|}{ Variable } & \multicolumn{7}{|c|}{ Terminals } \\
\cline { 3 - 8 } & & $\mathbf{1}$ & $\mathbf{2}$ & $\mathbf{3}$ & $\mathbf{4}$ & $\mathbf{5}$ & $\mathbf{6}$ \\
\hline \multirow{2}{*}{$\begin{array}{l}\text { BEB battery } \\
\text { capacity } \boldsymbol{k W h}\end{array}$} & 120 & 120 & 120 & 120 & 120 & 120 \\
\hline \multirow{2}{*}{$\begin{array}{l}\text { No of conductive } \\
\text { chargers }\end{array}$} & $\boldsymbol{H}$ & 2 & 2 & 1 & 2 & 1 & 1 \\
\cline { 2 - 8 } & $\boldsymbol{M}$ & 1 & 2 & 1 & 1 & 1 & 1 \\
\cline { 2 - 8 } $\begin{array}{l}\text { Total no. of } \\
\text { terminal chargers }\end{array}$ & & 6 & 5 & 3 & 4 & 3 & 3 \\
\hline $\begin{array}{l}\text { No of inductive } \\
\text { chargers }\end{array}$ & & 0 & 1 & 1 & 1 & 0 & 0 \\
\hline
\end{tabular}

Also, a comparative study is carried out between the priority and non-priority charging scenarios. In the nonpriority situation, the PSO algorithm is applied to find the optimal number of chargers for the Lagos BRT while 
considering the same capacity chargers for the six terminal in the transit network.

Table III: Simulation result for allocation of the non-priority chargers

\begin{tabular}{|l|c|c|c|c|c|c|}
\hline \multirow{2}{*}{ Variable } & \multicolumn{7}{|c|}{ Terminals } \\
\cline { 2 - 7 } & $\mathbf{1}$ & $\mathbf{2}$ & $\mathbf{3}$ & $\mathbf{4}$ & $\mathbf{5}$ & $\mathbf{6}$ \\
\hline $\begin{array}{l}\text { Capacity of conductive } \\
\text { chargers } \boldsymbol{k W h}\end{array}$ & 400 & 400 & 400 & 400 & 400 & 400 \\
\hline $\begin{array}{l}\text { Capacity of inductive } \\
\text { chargers } \boldsymbol{k W h}\end{array}$ & 0 & 500 & 500 & 500 & 0 & 0 \\
\hline BEB battery capacity $\boldsymbol{k W}$ & 120 & 120 & 120 & 120 & 120 & 120 \\
\hline No of inductive chargers & 0 & 1 & 1 & 1 & 0 & 0 \\
\hline No of conductive chargers & 5 & 1 & 3 & 1 & 3 & 4 \\
\hline Total no. of chargers & 5 & 2 & 4 & 2 & 3 & 4 \\
\hline
\end{tabular}

All the chargers considered in this scenario have the maximum capacity of $400 \mathrm{~kW}$. The simulation result for the allocation of the non-priority conductive chargers for the six terminal of the Lagos BRT electrification is shown in Table III. The optimal solution indicates that 17 conductive multiterminal chargers of the same capacity should be allocated to the transit network. The break-down per terminal is shown in Table III.

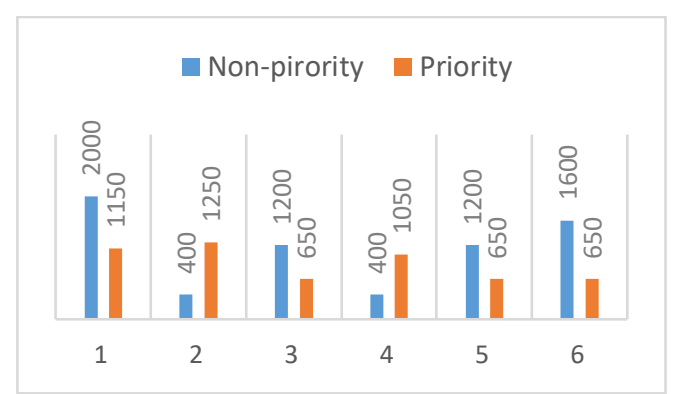

Fig. 5: kWh demand comparison for non-priority and priority charging per terminal

The chart that compares the total $\mathrm{kWh}$ capacity per terminal for both priority and non-priority charger is shown in fig. 5. the figure indicates that the total kwh capacity for priority charging strategy is $5,400 \mathrm{kwh}$ and $6,800 \mathrm{kwh}$ for non-priority; this has a difference of $1,400 \mathrm{kWh}$. Excluding capital costs, this indicates energy management and costsavings are achievable, especially on a long time scale.

\section{CONCLUSION AND Future RESEARCH}

The mathematical model for this research makes a small but significant approach, in that provision for charging priority that allocates chargers and capacity based on the state of charge on arrival at the terminal. When comparing the priority and non-priority cases, the result shows that savings can be achieved via energy management for the BEB transit network. The priority-charging present in this work is a cheap energy management system EMS that reduces energy usage in the short and long-term. This priority charging strategy can also reduce the investment in the power infrastructure because the model minimises the energy use from each terminal.

More effort is needed on the inductive charger modelling going forward as the existing approach is somewhat simplified. The priority-charging present in this work reduces energy usage in the short and long-term. However, more consideration is required of the network impacts that such investments in infrastructure would bring. This is especially true for public infrastructure in developing countries where the security of supply is often challenged. Future work will consider this aspect further and look to colocate distributed low carbon generation solutions with charging deployments.

\section{REFERENCE}

F. Agenda, "The Electrification of Transport," 2018. [Online]. Available:https://www.futureagenda.org/news/the electrification-of-transport-key-questions. [Accessed: 25-Jun2019].

W.P.Review, 'LagosPopulation'.[Online].Available: http://worldpopulationreview.com/world-cities/lagospopulation/.

A. A. Adeyanju and K. Manohar, "Effects of Vehicular Emission on Environmental Pollution in Lagos," vol. 5, no. 4, pp. 34-51, 2017.

4] D. Mobereola,"Africa's first BRT scheme,"SSATP Discuss.Pap. No. 9 Urban Transp.Ser.Available at http//www.ssatp.org/sites/ssatp/files/ publications/SSATPDiscussionPapers/DP09-Lagos-BRT.pdf, no. 9, p. 54, 2009.

[5] M. Mahmoud, R. Garnett, M. Ferguson, and P.Kanaroglou, 'Electric buses: A review of alternative powertrains', Renewable and Sustainable Energy Reviews, vol. 62. Elsevier Ltd, pp. 673684, 2016.

Zhou, B., Wu, Y., Zhou, B., Wang, R., Ke, W., Zhang, S. and Hao, J., 2016. Real-world performance of battery electric buses and their life-cycle benefits with respect to energy consumption and carbon dioxide emissions. Energy, 96, pp.603-613.

[7] G. Shlasky, "The advantages and challenges of electric buses," 2017.[Online].Available:https://www.linkedin.com/pulse/advan tages-challenges-electric-buses-gady-shlasky/. [Accessed: 12Sep-2019].

[8] Li, W., Li, Y., Deng, H. and Bao, L., 2018. Planning of electric public transport system under battery swap mode. Sustainability, 10(7), p.2528.

[9] Zheng, Dan, Fushuan Wen, and Jiansheng Huang. "Optimal planning of battery swap stations." (2012): 153-153.

[10] Mak, H.Y., Rong, Y. and Shen, Z.J.M., 2013. Infrastructure planning for electric vehicles with battery swapping. Management Science, 59(7), pp.1557-1575.

[11] Li, J.Q., 2016. Battery-electric transit bus developments and operations: A review. International Journal of Sustainable Transportation, 10(3), pp.157-169.

[12] Mohamed, M., Farag, H., El-Taweel, N. and Ferguson, M., 2017. Simulation of electric buses on a full transit network: Operational feasibility and grid impact analysis. Electric Power Systems Research, 142, pp.163-175.

[13] El-Taweel, N.A., Mohamed, M. and Farag, H.E., 2017, June. Optimal design of charging stations for electrified transit networks. In 2017 IEEE Transportation Electrification Conference and Expo (ITEC) (pp. 786-791). IEEE.

[14] Wei, R., Liu, X., Ou, Y. and Fayyaz, S.K., 2018. Optimising the spatio-temporal deployment of battery electric bus system. Journal of Transport Geography, 68, pp.160-168.

[15] Eberhart, R. and Kennedy, J., 1995, October. A new optimiser using particle swarm theory. In MHS'95. Proceedings of the Sixth International Symposium on Micro Machine and Human Science (pp. 39-43). Ieee.

[16] Kennedy, J. and Eberhart, R., 1995, November. Particle swarm optimisation. In Proceedings of ICNN'95-International Conference on Neural Networks (Vol. 4, pp. 1942-1948). IEEE.

[17] Y. D. Ko and Y. J. Jang, "The optimal system design of the online electric vehicle utilising wireless power transmission technology," IEEE Trans. Intell. Transp. Syst., vol. 14, no. 3, pp. $1255-1265,2013$ 